\section{The clot thickens}

\section{ByLev Osherovich, Senior Writer}

The suspected connection between coagulation and inflammation that leads to sepsis has been elucidated with the discovery of a signaling pathway that links the two. Dendritic cells turn out to be the key carriers of the coagulation-triggered inflammatory cue.

The notoriously difficult field of sepsis therapeutics may get a boost from a study in Nature that lays out one of several possible paths to the disease from blood clotting to inflammation. ${ }^{1}$ The findings tie together a number of earlier hints at how a runaway cycle of coagulation-driven immune activation leads to systemic inflammation and septic shock.

"It's long been known that there's a close link between the activation of coagulation and severe inflammatory response, but the molecular links were unknown," said group leader Wolfram Ruf, professor of immunology at The Scripps Research Institute and lead author on the paper. "We have found them."

Ruf's group identified the molecular mediators of sepsis using a mouse model in which injection of bacterial lipopolysaccharide (LPS) triggers lethal systemic coagulation and inflammation. Building on earlier work hinting at a connection between coagulation and sepsis, ${ }^{2}$ the team found that deletion of protease-activated receptor 1 (PAR1) or its inhibition by RWJ58259, a preclinical compound from Johnson \& Johnson Pharmaceutical Research \& Development, LLC, a subsidiary of Johnson \& Johnson, extended the survival of LPS-challenged mice compared with wild-type controls.

PAR1, which is normally activated by the clotting factor thrombin, is the target of the one marketed sepsis drug-Xigris drotrecogin alfa, a recombinant form of activated protein C, from Eli Lilly and Co.

Ruf's team then worked out the steps downstream of PAR1 that lead to inflammation and sepsis. Guided by previous reports linking PAR1 to the production of a sphingolipid called sphingosine 1-phosphate (S1P), the researchers tested mutants that lacked sphingosine kinase 1 (SphK1) and sphingosine 1-phosphate receptor 3 (S1P3) and found that these mutants also survived LPS challenge better than wild-type animals.

S1P has recently emerged as a major chemotactic cue for migrating cells. For example, its presence is required for $\mathrm{B}$ and $\mathrm{T}$ cells to exit the

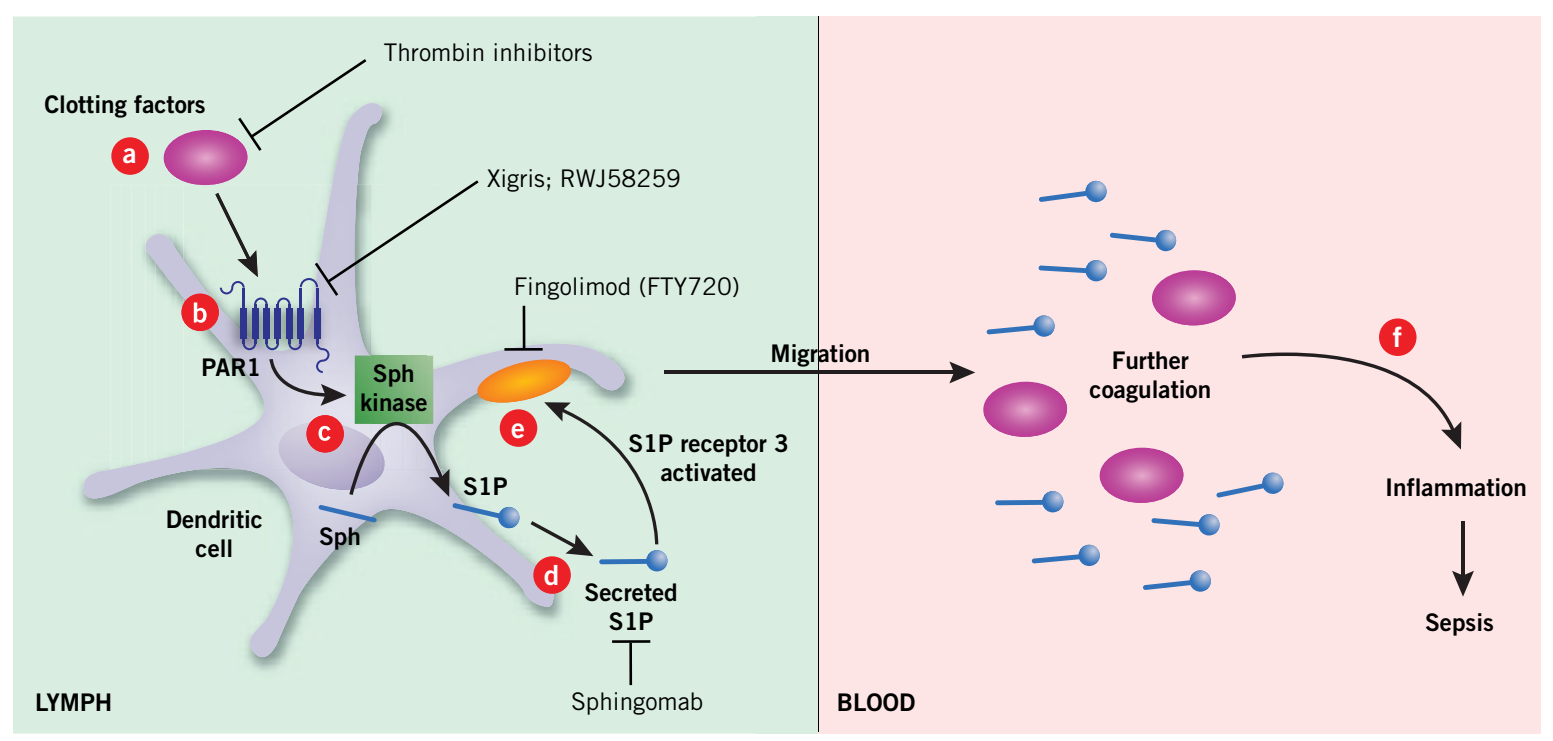

Figure 1. Dendritic cells connect coagulation to sepsis. Niessen et al. report that coagulation factors stimulate dendritic cells (DCs) to produce sphingosine 1-phosphate (S1P), initiating DC migration out of the lymph and into the blood and lungs, leading to sepsis.

These findings suggest the following model: coagulation factors such as thrombin [a] bind and activate protease-activated receptor 1 (PAR1), a G protein-coupled receptor on the cell surface [b]. In turn, PAR1 activates sphingosine (Sph) kinase [c], which phosphorylates membrane-associated sphingosine to make S1P, which is secreted [d]. S1P receptor 3 , a receptor on the DC surface [e], then senses S1P and triggers DC migration out of the lymph and into the blood (DCs move toward high concentrations of S1P). The escape of activated DCs into downstream vascular tissue, including the lungs, leads to further coagulation, inflammation [f] and sepsis.

A number of companies are developing compounds or have marketed drugs that could disrupt steps in this pathway (see article for details). 
lymph nodes after being activated by exposure to antigens. ${ }^{3}$

Experiments with various combinations of these mutations and compounds that agonized or antagonized PAR1 and S1P3 allowed Ruf's team to put the pathway into order and showed that a coagulation-triggered S1P spike sets off the cytokine storm that leads to sepsis (see Figure 1, "Dendritic cells connect coagulation to sepsis").

Perhaps the most surprising finding in Ruf's paper is that dendritic cells (DCs) are the cells responsible for the PAR1 and S1P modulation of sepsis. Wild-type DCs alone could reconstitute sensitivity to sepsis in an otherwise resistant mutant animal, suggesting that these migratory innate immune cells transmit the S1P sepsis signal.

Excessive DC activity has previously been linked to sepsis. "The dendritic cells are very active in releasing exosomes or membrane components to communicate with T cells," Ruf said. "In sepsis, the problem is that this shedding occurs in the wrong location," such as the lungs.

Instead of leaking out into the postlymphatic vascular system and causing sepsis, DCs lacking PAR1 or S1P signaling appear to remain in the lymph nodes. This suggests that disrupting the pathway does not compromise the normal acquired immune response in which DCs deliver antigens to the lymph nodes to prime B and T cells. "The most impressive finding for us was that as soon as we rebalanced the systemic inflammatory process, we could localize the inflammation in lymph nodes," said Ruf.

These findings open new angles for drug makers trying to treat systemic inflammation before it leads to full-blown sepsis.

One key challenge has been that the bacterial infections that usually lead to sepsis require a vigorous immune response. Thus, indiscriminate immunosuppressants are a bad idea. "There's been a long history of trying to block inflammatory cytokines, but all of these things have failed in the clinic" for sepsis, Ruf told SciBX.

Instead of treating late-stage sepsis symptoms like elevated interleukin-1 $\beta$, blocking pathogen-derived molecules or host signaling molecules that trigger septic shock may be a more promising approach.

"Sepsis has killed nearly as many companies as it has people," said Alan Solinger, VP of clinical immunology at Xoma Ltd. The biotech survived its foray into sepsis when its mAb against LPS (the bacterial molecule also known as endotoxin) failed its third Phase III trial in 2000.

Solinger said Ruf's paper was a promising development. "After all these years of failure, when a target comes up, people are interested."

"What's really novel about this paper is that PAR1 and sphingolipids could be targets for sepsis treatment," said Roger Sabbadini, VP and CSO of Lpath Inc. The company is targeting S1P directly with its Sphingomab. The $\mathrm{mAb}$ is in Phase I testing for cancer and age-related macular degeneration.

Sabbadini told SciBX Ruf's paper suggests that "Sphingomab could also be suitable for the treatment of septic shock."

Yann Echelard, VP of research at GTC Biotherapeutics Inc., was less sanguine about the idea of PAR1 and S1P signaling as stand-alone targets for sepsis. He doubted that "single-agent modulation of the PAR1 pathway could on its own overturn sepsis," citing the complex immunomodulatory effects of coagulation drugs such as antithrombin, which can actually exacerbate the symptoms when administered together with heparin (a standard combination for nonseptic patients). ${ }^{4}$

GTC's ATryn, a recombinant antithrombin, is in Phase III testing for disseminated intravascular coagulation, which is a complication of severe sepsis. The product is partnered with Leo Pharma A/S.

"It is very possible that drugs that directly affect the PAR1 pathway will synergize with ATryn," said Echelard. "However, only combined animal studies are likely to occur in the near future."

One immunomodulatory compound in the PAR1 and S1P pathway is FTY720 (also known as fingolimod), an S1P receptor inhibitor being developed by Novartis AG and Mitsubishi Tanabe Pharma Corp. FTY720 is in Phase III trials to treat multiple sclerosis. Earlier Phase III trials of FTY720 as a kidney transplant immunosuppressant failed. ${ }^{5}$

FTY720 also has characteristics that may limit its potential in sepsis. In preclinical studies, the molecule was shown to block a number of different S1P receptors and shut down $\mathrm{B}$ and $\mathrm{T}$ cell migration, leading to lymphopenia. ${ }^{3}$ This may make it a good immunosuppressant but potentially bad for septic patients trying to mount a robust immune response against pathogens.

"Ideally, one would want to develop a functional S1P3 antagonist" that would not interfere with other S1P receptors, Sabbadini told SciBX.

Julie Morrow, a Novartis spokesperson, said Ruf's research is "really interesting, but it is a bit too early to speculate" about using FTY720 to treat sepsis.

\section{REFERENCES}

1. Niessen, F. et al. Nature; published online Feb. 27, 2008; doi: 10.1038 /nature06663

Contact: Wolfram Ruf, The Scripps Research Institute, La Jolla, Calif. e-mail: ruf@scripps.edu

2. Ruf, W. J. Throm. Haemost. 3, 1912-1914 (2005)

3. Schwab, S.R. \& Cyster, J.G. Nat. Immunol. 8, 1295-1301 (2007)

4. Wiedermann, C.J. Crit. Care 10, 209 (2006)

5. Westhoff, T.H. et al. Nephrol. Dial. Transplant. 22, 2354-2358 (2007)

\section{COMPANIES AND INSTITUTIONS MENTIONED}

Eli Lilly and Co. (NYSE:LLY), Indianapolis, Ind.

GTC Biotherapeutics Inc. (NASDAQ:GTCB), Framingham, Mass.

Johnson \& Johnson (NYSE:JNJ), New Brunswick, N.J.

Johnson \& Johnson Pharmaceutical Research \& Development LLC, Spring House, $\mathrm{Pa}$.

Leo Pharma A/S, Ballerup, Denmark

Lpath Inc. (OTCBB:LPTN), San Diego, Calif.

Mitsubishi Tanabe Pharma Corp. (Tokyo:4508; Osaka:4508), Osaka,

Japan

Novartis AG (NYSE:NVS; SWX:NOVN), Basel, Switzerland

The Scripps Research Institute, La Jolla, Calif.

Xoma Ltd. (NASDAQ:XOMA), Berkeley, Calif. 